\title{
A hybrid approach for personalized news recommendation in a mobility scenario using long-short user interest
}

\author{
Paula Viana \\ INESC TEC, Campus da FEUP Rua Dr. Roberto Frias, \\ Porto, 4200 - 465, Portugal, \\ Polytechnic of Porto, School of Engineering, Rua Dr. António Bernardino de Almeida, \\ Porto, 4249 - 015, Portugal, \\ paula.viana@inesctec.pt \\ Márcio Soares \\ INESC TEC, Campus da FEUP Rua Dr. Roberto Frias, \\ Porto, 4200 - 465, Portugal \\ mmsoares@inesctec.pt \\ Received (Day Month Year) \\ Revised (Day Month Year) \\ Accepted (Day Month Year)
}

\begin{abstract}
Access to information has been democratized and made easier with the huge evolution in communication systems and electronic devices. This has an impact on everyday life, as users are requesting and generating information in a rate never seen before, introducing also new challenges for content owners. In the news domain, this scenario is even more obvious given the large amount of news articles produced daily and their ephemeral timelife. Consumption of news has also been impacted by the ever more powerful and ubiquitous mobile devices. Recommendation systems are often used to help content providers on filtering critical information, key events and meaningful items that might be of interest to a reader. In a mobility scenario, context-aware approaches may improve the user satisfaction and his loyalty to a service.

In this paper, a news recommendation system, which combines content-based and georeferenced techniques in a mobility scenario, is proposed. Taking into account the volatility of the information, short-term and long-term user profiles are considered and implicitly built based on user actions that enable inferring different levels of interest. Results have been tested with a population of volunteers and results indicate that the quality of the recommendation system is acknowledged by the users.
\end{abstract}

Keywords: News recommendation system; geolocation; mobile application; hybrid recommender.

\section{Introduction}

Online news reading has become very popular as the web provides access to news articles from millions of sources around the world.

People typically read news to know and understand what happened, what is happening and will happen in a town, region, country, or in the world. This information demand presents itself a key challenge for news providers on helping users finding, fast and accurately, those news which are of interest for them. Manually searching and analysing 
the available news articles, to select those considered interesting, is not feasible within the time constrains common for most users. In this scenario, recommendation systems stand out as a possible solution to help newspaper readers on the selection of content that best fits his/her preferences. Additionally, assisting tools that guide the reader in the content selection process can contribute to increase his loyalty to a specific service/product and thereby contribute to improved cost-effectiveness for the service provider.

The dissemination of mobile devices like smartphones and tablets on everyday activities introduced an additional shift on the reading habits, from the online reading paradigm in the computer screen to everywhere, anytime and from any mobile device. Due to the growing use of mobile devices, research on recommendation approaches suitable for this emergent scenario has been recently addressed by the academic community ${ }^{9,37,41}$. However, despite this increasing awareness, the field of mobile news articles recommendations is still fairly unexplored.

Mobile and ubiquitous computing provide extended means to sense data and enhance recommendations that can deliver interesting topics to the user, in any place, at any time, for example, while traveling on a business trip or on holidays. As they can provide information on the geographic location of the reader, this information can also be used by recommendation systems to provide news articles with the same geolocation.

In this paper, a hybrid recommendation system for news in a mobile environment is presented. The implemented system was integrated in a mobile application of the Portuguese newspaper Público* in the context of the project Pglobal. Pglobal is a new concept of editorial content aggregation that joins in the same platform a network of newspapers, universities and users from various Portuguese-speaking countries. The goal is to build a community of readers who have in common the language and to create or consolidate a paywall for collecting digital content and subscriptions and explore the information business in the international Portuguese-speaking market.

The implemented hybrid recommendation system combines content-based and georeferenced recommendation methodologies. Using these approaches, recommendations are generated based on a user's profile created implicitly by monitoring the user interactions with the mobile application, in particular the news that he reads, shares on social networks or that he Likes. Additionally, the geolocation of the mobile device is also implicitly registered.

Since the reader's interests may change over time, the process of observing his reading habits, to enable adapting the output accordingly, is performed by maintaining a short-term and a long-term profile. Using the user's two profiles, the system is able to recommend two types of items: (1) items similar with the ones with which the user has recently interacted with, associated to the short-term profile; (2) items containing the terms/tags most read by the user, associated to the long-term profile. Additionally, the geolocation of the mobile device is also used in order to recommend news articles related to the same location. The final recommendation list is obtained by combining recommendations provided by each of the approaches.

\footnotetext{
*http://www.publico.pt
} 

interest

The solution was evaluated by a group of volunteers and results indicate that the quality of the recommendations is acknowledged by those test users.

The rest of this paper is structured as follows. In Section 2, we survey the available literature on various state-of-the-art news recommender system approaches. Section 3 gives an overview of the overall system architecture and component interaction. The methodologies used for extracting information from the news content, to create user profiles and to recommend items are presented. In Section 4 the propose solution is evaluated by performing a user study. The results are presented and discussed in detail in Section 5. Finally, in Section 6 some conclusions are taken and future work is highlighted.

\section{Related Work}

Recommender systems are systems with the ability of providing suggestions or directing a person to a service, product or content, that has a potential of interest among a number of different alternatives.

Although the first recommendation systems date from the late ' 70 s, only in the early '90s the first commercial applications of this type of systems were deployed. Currently, highly rated internet sites as Amazon ${ }^{\dagger}$, eBay ${ }^{\star}$ or YouTube ${ }^{\S}$ use recommender systems as part of their services.

Beyond these examples, recommender systems also apply on numerous other domains including books ${ }^{11}$, movies and TV programs ${ }^{45,46}$, music ${ }^{22,31}$, news articles ${ }^{6,49}$, and so forth

In this section we overview the most relevant recommendation techniques that have been proposed in the news field. Existing experiences on mobile environments are also addressed.

\subsection{Methods for collecting data and building user profiles}

Over the years, recommendation of online news articles has become an area of great interest. Large newsfeed portals, such as Google News, and Yahoo! News, provide personalized news recommendation services for a large amount of online users.

The task of recommending news articles based on the user's preferences can be conducted using distinct methodologies. Approaches adopting content-based and collaborative filtering are widely used by existing news recommender systems. Alternatively to using one specific approach individually, hybrid methodologies resulting from the combination of multiple methodologies have also been explored.

The provision of personalized recommendations requires that the system knows something about the user. Recommender system must create and maintain a user model or user profile that contains user's preferences. For that, information may be collected explicitly, through direct and conscious user intervention, or implicitly, through agents that monitor user actions or activity.

\footnotetext{
${ }^{\dagger}$ http://www.amazon.com

http://www.ebay.com

$\S$ http://www.youtube.com
} 
Besides specific information on user preferences over a set of pre-defined product alternatives that may be requested, explicit feedback is often provided in the form of rating actions. In the DailyLearner ${ }^{3}$ portal, users explicitly rate news stories classifying each as interesting or not interesting. Claypool et al. ${ }^{10}$ uses an alternative approach where users are allowed to select, from a list of newspaper categories (Movies, Food, Health, etc.), the ones that best fit their preferences. Similarly, Ahn et al. ${ }^{2}$ proposes an open and editable user model for personalizing news items with an explicit profile component that enables users to view the list of keywords stored in their individual profiles and add or delete keywords.

Most extensively used sources for implicit feedback include reading time, click behavior, scrolling, etc. Lai $e t$ al. ${ }^{29}$ propose an approach that builds newsreaders profiles from their time reading behavior. The basic assumption of the authors is that a reader will spend a reasonable amount of time reading an interesting article. Other studies have also shown mouse clicks ${ }^{12}$ and scrolling ${ }^{26}$ on the news to be useful sources of implicit feedback for predicting user's preferences.

Profiles that can be modified or improved are considered dynamic, in contrast to static profiles that maintain the same information overtime (e.g. personal background, personality or demographic information). Dynamic profiles, that take time into consideration, may discriminate short-term and long-term interests ${ }^{4,15}$. Usually, short-term profiles are constructed by considering only the most recent user preferences. In contrast, a long-term profile is based on the sum of the data collected since the user has begun to be monitored. Focused on the issue of how users' interests change over time Liu et al. ${ }^{34}$ conducted a large-scale analysis of anonymized Google News users click logs in order to measure the stability of users' news interests. The log analysis demonstrated, indeed, variations in users' interests over time and shows that these variations can be explored to improve the quality of news recommendations. Similarly, in order to demonstrate the relevance of the user's reading time evolution $\mathrm{Li}$ et al. ${ }^{33}$ propose a news recommender system in which the long-term and short-term reading preferences of users are seamlessly integrated when recommending news items to individual online users. Results show promising performance compared with other existing methods.

\subsection{Recommendation approaches in the news domain}

\subsubsection{Content-based recommendation systems}

According to a definition provided by Lops et al. ${ }^{35}$, content-based recommendation systems try to recommend items similar to those a given user has liked in the past. In content-based approaches to news recommending, the main process consists in matching up the attributes of a user profile, in which preferences and interests are stored, with the attributes of news content, in order to recommend to the user new interesting articles.

News content is often represented using a vector space model (e.g., TF-IDF ${ }^{7}$ ) or topic distributions obtained by language models (e.g., LDA $^{18}$ ) and specific similarity measurements are adopted to evaluate the relatedness between articles and user profiles. 
NewsDude $^{3}$ was one of the earlier representatives of content-based news recommendation systems. The approach consisted on presenting news stories to the users, who then rated the articles according to whether they were considered interesting or not. The user profile built on this information was then compared with content of other available articles to generate personalized recommendations. Another content-based solution is Newsjunkie ${ }^{14}$ that analyses the dynamics of novelty in recommendations, and personalized news stories are generated by identifying the novelty of news' topics.

Lops et al. ${ }^{35}$ proposes a method that uses a selection of parameters to estimate news relatedness. These parameters include relevance, novelty, connectivity, and transition smoothness. Recommendations are estimated by finding the news with greater relatedness to the news articles in the users' profiles.

A content-based approach with some new contributions is presented in Blaco et al. ${ }^{5}$. In this work, the authors propose to enhance effectiveness of news recommender systems by adding, to each recommendation, an explanatory statement to help the user better understanding if, and why, the item can be of interest. Experiments show that news recommender systems can greatly benefit from using explanation modules.

In the last few years, some content-based approaches start focusing on exploring news semantic concepts in order to improve recommendation's quality. A news item often contains key concepts that capture the semantic context of the article. Recommenders that focus on the key concepts might produce faster and more accurate recommendations than the simple content-based recommenders, since they don't need to consider all words and, unlike words, concepts are not ambiguous. Several examples of semantic-based approaches are discussed in Frasincar et al. ${ }^{13}$, Goosen et al. ${ }^{20}$, IJntema et al. ${ }^{23}$, Schouten et al. ${ }^{43}$, Solomou et al. ${ }^{47}$.

\subsubsection{Collaborative filtering recommendation systems}

Collaborative recommendation systems consider that users with similar reading behaviours in the past will usually have similar preferences about news articles in the future. Grouplens ${ }^{42}$ was the earlier representative of a collaborative filtering approach in the news domain. In Grouplens, users rate articles after reading them and this information is used to correlate users with similar scores. Afterwards, based on ratings from similar users, the system predicts how well users will like new articles.

Although collaborative filtering is one of most mature and commonly implemented recommendation approaches, it is only able to effectively capture user's behaviors in cases where overlap in historical consumption among users is relatively high and the content universe is nearly static. In the news domain, however, the life of the content is, in general, ephemeral and the number of stories and their content is constantly updated. In order to overcome these specific limitations, some refined collaborative approaches have been studied. Gao et al. ${ }^{15}$ proposes an online collaborative algorithm based on context trees that can provide high-quality news recommendations to anonymous visitors based on the current user browsing behaviour. Google News ${ }^{12}$ is another popular example of a news 
articles recommender based on collaborative filtering. Google News is an online news portal that aggregates news articles from thousands of sources, grouping them to the users, according to their personal interests. The recommendation approach is based on implicit user and community feedback represented by the user click history. More recently, Xiao et $a l^{50}$ proposes a time-ordered collaborative filtering recommendation algorithm (TOCF), which takes the time sequence characteristic of user behaviors into account. Moreover, a new method to compute the similarity among different users, named time-dependent similarity, is proposed. Experimental results show that the developed algorithm performs better than traditional collaborative filtering algorithms when they are used to predict the next news article the user will read.

\subsubsection{Hybrid recommendation systems}

Content-based and collaborative filtering can provide meaningful recommendations but each of the approaches has, however, some disadvantages ${ }^{46}$. In order to improve the overall performance, hybrid approaches to news recommendations have also been explored. Representative examples include Claypool et al. ${ }^{10}$, García et al. ${ }^{16}$, Jonnalagedda and Gauch $^{25}$.

P-Tango ${ }^{10}$ was one of the first news hybrid recommendation approaches that recommend news items by combining content-based and collaborative filtering using a weighted average function. García et al. ${ }^{16}$ proposes an adaptive hybrid recommender. The term adaptive hereby refers to the fact that the system supports more than one collaborative recommender algorithm. Jonnalagedda and $\mathrm{Gauch}^{25}$ presents a hybrid personalized news recommender system that recommends interesting news articles to the user using the micro-blogging service Twitter.

\subsection{Mobile news recommendation systems}

Technical advances towards ever more powerful mobile devices and their fast market penetration have been happening quite fast. Therefore, mobile applications - and ubiquitous applications in general - constitute a promising application domain for different types of personalization and recommendation.

Although news recommendation has been significantly explored in recent years, most research was conducted considering only user interaction with a computer screen.

Approaches directly related to mobile devices have only recently started to appear. Representative examples include Gao et al. ${ }^{15}$, Lee et al. ${ }^{30}$, Shapira et $a l .{ }^{44}$, Tavakolifard and Gulla ${ }^{48}$.

Gao et al. ${ }^{15}$ presents InfoSlim, a personalized news recommendation system on mobile devices that exploits information on the content the user has read to find what user is really interested in.

MONERS $^{30}$ presents a mobile web news recommendation system, which determines users' preferences for news articles based on the importance of the news event, the recency of the article, changes in users' preferences, user segments and article preferences. 

interest

Shapira et $a l .{ }^{44}$ proposes ePaper, a research prototype system of a personalized newspaper on a mobile reading device which aggregates content from several different news providers. The ePaper classifies the news items according to concepts from a news domain ontology, and delivers an electronic newspaper to each subscriber according to the user's profile.

The additionally that mobile devices bring into recommendation systems is that they enable knowing the user's current location. Georeferenced-based recommenders, that combine information on user's location with information about user's interests, can be used to provide e.g. the vicinity of restaurants, entertainment and shopping information, etc. Despite the existence of some georeferenced recommendation systems ${ }^{21,36,51}$, mobile news recommendations based on geolocation remains an unexplored area.

Tavakolifard and Gulla ${ }^{48}$ describes one of the few mobile news recommendation systems using this functionality. This proposal describes a personalized mobile news recommender system which combines various methods of recommendation, particularly designed for the domain of news articles. It considers a variety of factors including freshness and users' preferences as the main recommendation targets. According to the authors, the information about location is mainly used to alleviate cold-start problems.

More recently, Garrido et al. ${ }^{19}$ presents an approach to develop a personalized news recommendation system as an application for mobile phones that takes into account the geolocation of the user. The developed recommendation engine is designed to identify name entities corresponding to locations that are mentioned in the textual content. It then expands and filters the locations to obtain all the places whose description matches the named entity in the news text. Based on a built news's geographical graph, the system makes recommendations to users depending on their current location.

The solution being described in this paper uses the concept of geolocation in three domains: the current location of the reader obtained by the device sensors; the identification of the original source of a news item and respective country; the identification of the place the text refers to through geo-tagged information attached to the content.

\section{Recommendation system implementation}

Figure 1 depicts the architecture of the developed system. Four main blocks can be identified: Pglobal News Provider, Mobile Device, Device logs Server and Recommendation System Core.

- Pglobal News Provider is responsible for aggregating and providing, news articles from multiple Portuguese language newspaper sources to the recommendation system and to the mobile device. Periodically, the recommendation system module uploads the latest news articles and processes and stores them for future use.

- The Mobile Device runs the news App responsible for presenting generic news and recommendations to the user.

- The Device Logs Server is responsible for storing device logs. The logs information contains data about the user's interactions with the App (the identification of the type of interaction and the date when it was performed). A representative example of 
possible interactions includes sharing or reading news. The logs information contains also the device ID as well as the code name of the country where the device is located. Periodically, the recommendation system module uploads device logs information to process and create or update devices' profiles.

- The Recommendation Core is the main component of the system. This module has, as input, previously processed news and devices' profiles. All recommendations are periodically generated in offline mode and the final produced recommendations are sent to the Pglobal repository in order to be made available to a mobile device.

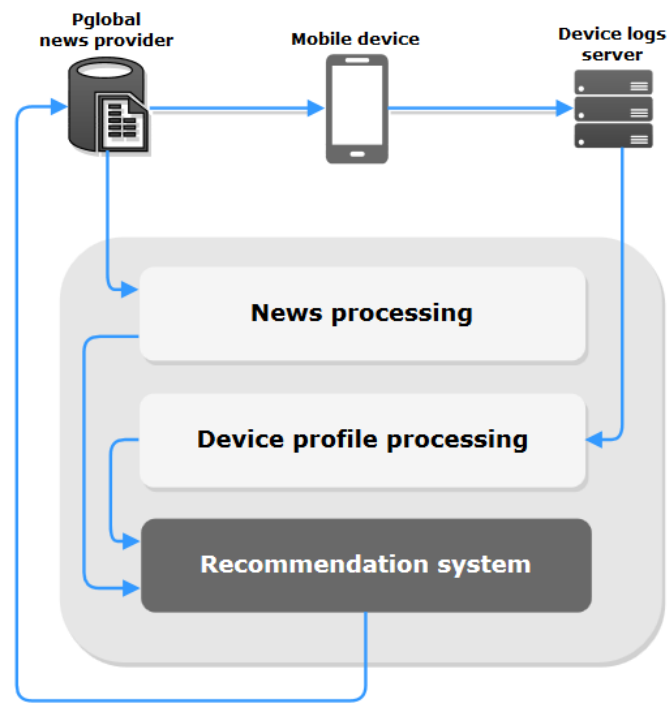

Fig. 1. News recommendation system architecture

\subsection{News processing}

News agencies around the world produce a large number of information every day. Text, obeying to natural language syntactic rules, produces a huge number of elements necessary for people to have a perfect understanding of the story. However, most of the content can be discarded when being processed by machine learning systems. This process simplification improves the efficiency of the systems since it enables focusing the analysis on the useful information only.

In order to increase the efficiency of our recommendation system, a set of operations is performed on the news content. Figure 2 represents the dataflow of this news processing procedure that includes three main modules: the News Pre-Processing, the Data Clustering and the Similarity modules.

As a first step and periodically, the News Pre-Processing Module requests the latest news articles available in the system and applies a set of text mining techniques in order to reduce the news word space. The new content resulting from this process is stored in the system database. 

interest

After this initial processing phase, the Clustering module performs the operations needed to organize and group all existent news according to their topics.

Finally, the similarities between all the news, within each of the created clusters, are computed and resulting values are also stored in the system database in order to update the relationship map between all news.

Each of these steps is described in more detail in the following sections.

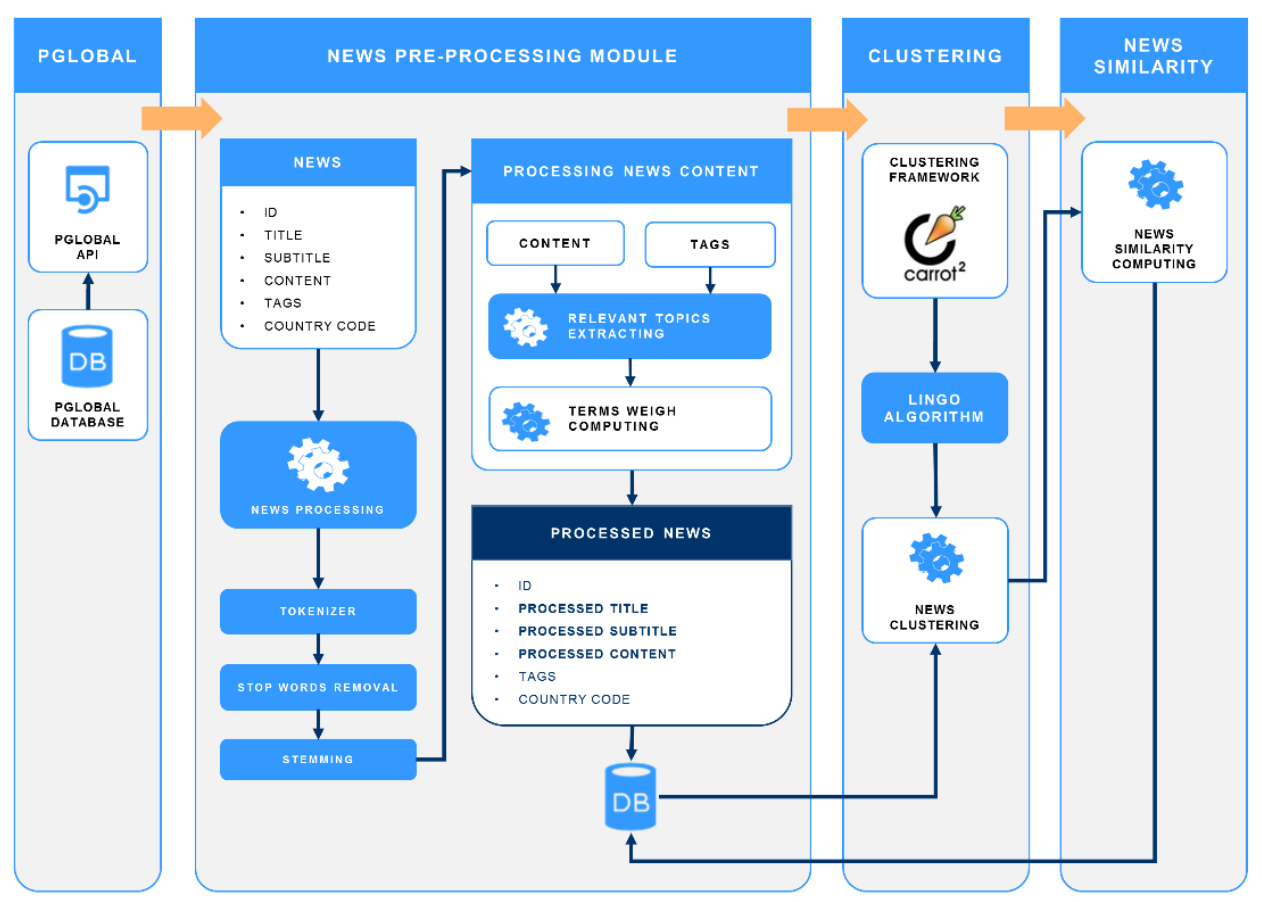

Fig. 2. Dataflow of the news processing procedure

\subsubsection{News Pre-Processing}

In order to extract key information, the system first pre-processes the news article. The first step is to split the title and subtitle into tokens. This is an important prerequisite for various linguistics tasks. In a second step, all common non informative words, defined in literature as stop words, are removed from these fields. The next step involves stemming text, reducing inflected words to their stem (or root) word.

For the main content of the news, despite stemming and removing stop words enables simplifying the text by reducing the number of textual elements, news articles can still be very long. In those situations, the remaining text can still be too complex in order to capture the key topics of the text. To retain just the essential information, a filtering process, using the metadata elements associated with the news, is performed. The metadata elements may vary from provider to provider but a set of standard elements is often used:

- $\quad$ title - title of news; 
- $\quad$ subtitle - subtitle of news usually introducing key/relevant information;

- content - the main text of the news;

- tags - tags assigned to a news article that capture key concepts and main topics.

In a first step, tags are used to remove all words from the main text that do not match these topics.

After this process, the weight of all the remaining words, in all metadata fields, is estimated. This weight shall represent the importance of that term and is estimated combining the number of times that the term occurs, together with the relevance weight of the field where it occurs: words appearing in the title of a news story will have a greater weight compared to a word that can occur many times, but only in the main text of the news. The application of this metric produces a vector where each word in the news article has a weight associated that is estimated according to Equation (1)

$$
\text { TermWeight }=\left(\frac{\text { Termoccurences }}{\text { TotalTerms }}\right) \max (\text { TermBoost })
$$

where TermOccurences is the number of term occurrences in all news article fields; TotalTerms is the total number of terms and TermBoost is the metadata field relevance weight where the term occurs. Given that the mobile device has limited space for showing the information, the App will, at a first stage, show a partial view of the news. The relative importance of each of the fields reflects this situation: Title is attributed an impact of 4 , Subtitle of 2, while Content is classified as a 1. If the term occurs simultaneously in the three metadata fields, the max weight is considered.

\subsubsection{Clustering}

Depending on the news source, dozens of new items can be introduced in the system daily. Estimating the similarity of all these new items with those already stored in the system can result in a very time consuming computational task. Grouping articles according to their content in a set of clusters that contain similar items can contribute to decrease the calculation time of similarity.

Clustering is a process of creating groups (clusters) of similar objects from a given set of inputs. When applied to news, clustering can be perceived as a way of organising articles into a number of easily identifiable thematic topics. Two main requirements can be defined a priori: (1) overlapping clusters should be possible - since news usually cover multiple topics, it is important to avoid confining each article to only one cluster; (2) efficiency should not be compromised by the clustering process - given that the process will be used as part of an on-line system and a large number of news can be introduced daily, with a very short lifetime, it is crucial that the clustering algorithm does not introduce noticeable delay and it is implemented as an incremental process.

The implemented clustering process is supported by the Carrot 2 Framework ${ }^{* *}$. This framework can automatically organize small collections of documents, e.g. search results, into thematic categories. From the list of available approaches, the Lingo algorithm ${ }^{38}$ was

** http://project.carrot2.org/index.html 

interest

selected given that, according to the framework documentation ${ }^{39}$, it is the algorithm that best fits the news domain.

Lingo is based on singular value decomposition (SVD) of a term frequency matrix and an a priori process of cluster labelling. The general idea behind Lingo algorithm is to first find meaningful and humanly understandable descriptions of clusters (labels), and then, based on those descriptions, determine their content. Careful selection of cluster labels is then crucial - the algorithm must ensure that the labels differ significantly from each other but, at the same time, cover most of the topics in the input collection.

Figure 3 shows an example of the clusters obtained using the Carrot 2 framework. In this example it is possible to visualise the labels associated to each cluster, identifying its main subject. Since the cluster labels are humanly identifiable, it is possible to visualise the overall relationship between the clusters.

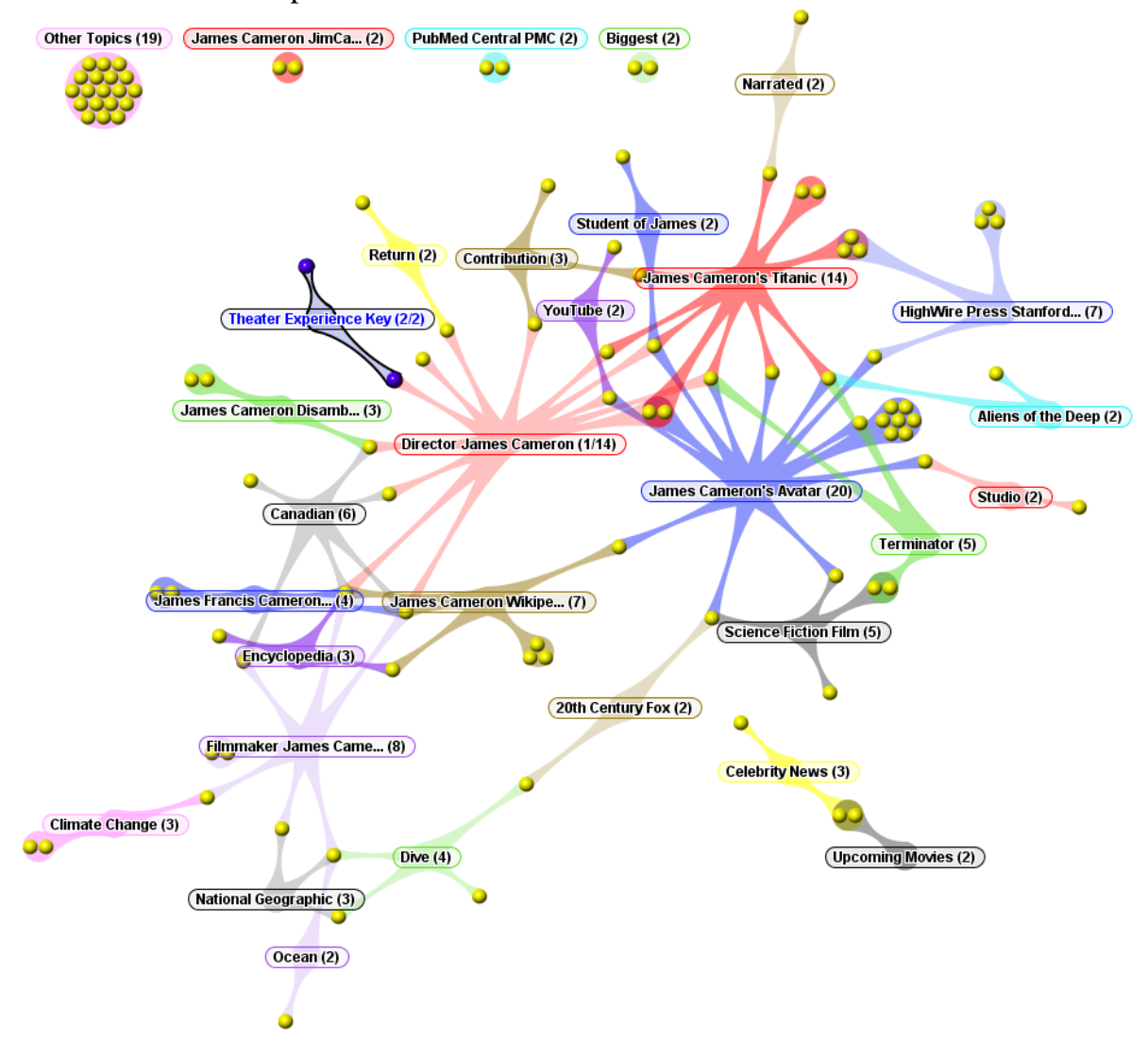

Fig. 3. Result of a clustering process using the framework Carrot2

Figure 4 illustrates the result of applying the clustering process to news in the Pglobal system. Each of these clusters has a set of news that are clearly related to the identified cluster topic. 


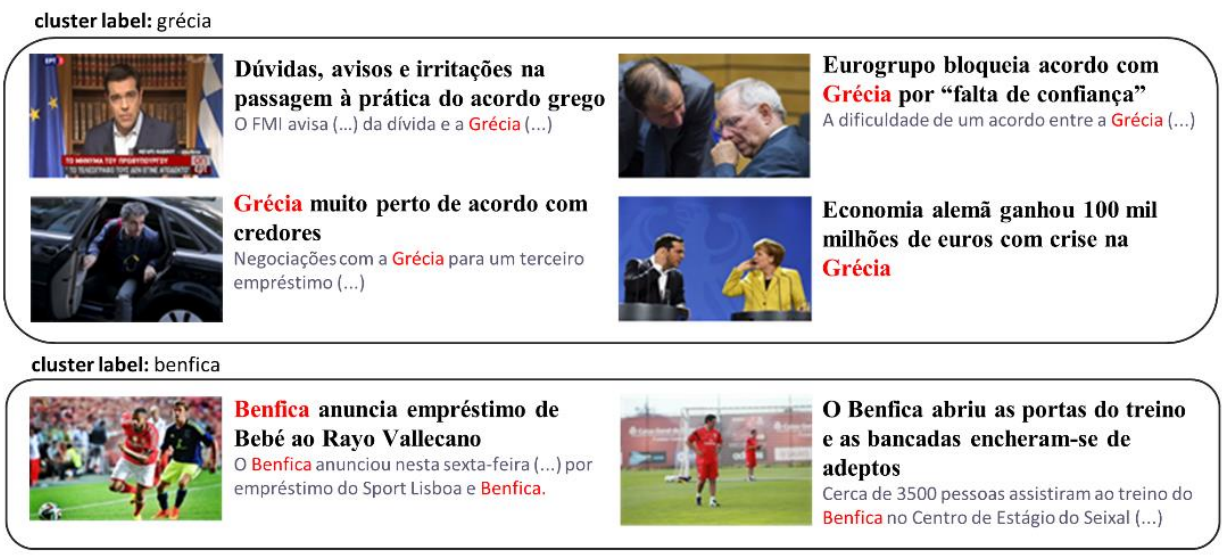

Fig. 4. Clustering of news in the Pglobal system

\subsubsection{Similarity computation}

Any content-based recommender system tries to estimate how similar items are. By first defining clusters, we can lower the time needed for similarity estimation. Instead of computing the similarity between all existing items, our system only computes the similarities between items belonging to the same cluster. This methodology avoids calculating the similarity between items that do not have any content in common, increasing the system efficiency. The similarity between items inside each cluster is estimated using the items' weight terms vectors by applying the Cosine similarity measure.

\subsection{Device Profile}

Users' profiles are the core of many recommendation systems ${ }^{1,8,25}$. These profiles may consist in a number of different types of information. For the online newspaper domain, the user profile might contain the type of news or categories the user likes to read. This information can be collected either explicitly or implicitly.

A key issue for news recommendation is how to capture each user's reading interests according to his/her historical consumption. Additionally, it is important to take into account the evolution of users' reading interests. According to Li et al. ${ }^{32}$, the topics a user is interested in, can be relatively stable or vary slightly in a long-term perspective, whereas the content accessed by the user might change along different short-term perspectives.

In order to consider the evolution of users' reading preferences, a user short-term and long-term profile was implemented.

To reduce the dependency on the will of the user to interact with the systems other than to read news, the system does not assume any direct user feedback on the articles present to him. In our implementation, the user's profile is constructed by collecting information implicitly, from the observation of the his actions. All the collected information is stored in a $\log$ file that is periodically sent to a web server that processes and stores this information. 

interest

Typically, profile construction and maintenance relies on the history of logged-in users. This might cause potential privacy issues to the users and prevent them from using the system. Our work departs from this restriction and considers only a device profile, which does not require the user login. However, given that mobile devices are usually personal and not-transferable, the collected information is expected to appropriately describe the user. The shortcoming of this approach is that if a user changes the device, a user profile synchronization will not be possible unless a login process is performed.

Implicit information results from three types of users' interaction with the mobile application:

- $\quad$ Read - The read action is registered when a user keeps in the same news screen for a pre-defined period of time - for example, more than 10 seconds.

According to Kelly ${ }^{27}$ it should be noted that the amount of time that an object is displayed does not necessarily correspond to the amount of time that the object is viewed. However, studies like the one presented in Konstan el al. ${ }^{28}$ claim that predictions based on time spent reading are nearly as accurate as predictions based on explicit numerical ratings, which is a good indicator for using this approach.

In order to capture implicit feedback about the user's interest in a news article, other approaches can be considered. Representative examples include mouse $\mathrm{click}^{24}$, scrolling ${ }^{7}$ or eye tracking ${ }^{40}$. However, each of these approaches has some limitations ${ }^{13}$ : using scrolling as an indication of interest may fail, for example, in short texts as scrolling may not be necessary. Additionally, scroll speed should also be considered because, if a user scrolls down quickly on a page without focused reading, the action should instead be interpreted as a sign of lack of interest.

- Like - This action corresponds to a Facebook like that a user gives to a news article.

- Share - This action corresponds to sharing a news article on an available social network, namely, Facebook, Twitter and Google+.

\subsubsection{Long-term profile}

The Long-term profile is used to model the user's general topics interest and is created based on the news that a user reads over time. For that, the system collects the tags associated to these news and the profile is created by considering the number of times that a specific tag has been read by a user. For example, if a user reads 10 news containing the tag "football" that tag will have a weight of 10 .

The main objective of this profile is to be able to collect full life information while still keeping the computation costs for the recommendation algorithm within feasible limits.

\subsubsection{Short-term profile}

The Short-term profile tries to capture recent or seasonal interests, as very popular news. The thought behind this type of profiles is that interest is driven by curiosity or short-term happenings and can quickly become uninteresting. This aspect is also relevant in a mobility scenario, as users will probably modify their reading habits during short stays in other locations. 
In this work, the short-term profile is based on the set of recent news with which the user has interacted (read, like or share) with and that were still not considered and processed by the recommendation system. Given that the data set (news articles) for this scenario is shorter, all the fields contained in the news will be processed and used by the recommendation algorithm to provide similar content.

\subsection{News recommendation system approach}

The main purpose of selecting a personalised set of news to a given reader, can be formalised by the definition presented in Adomavicius and Tuzhilin ${ }^{1}$ and formulated as:

$$
s: U \times A \rightarrow V
$$

where $s$ represents the utility function, $U=\left\{u_{1}, \ldots u_{n}\right\}$ the set of users, $A=\left\{a_{1} \ldots a_{n}\right\}$ a set of articles in the repository and $V$ an ordered set of values representing the rating or preference of a user for an article. The recommendation problem can be formulated as selecting an unknown item $a^{\prime} \in A$ for each user $u \in U$ that maximizes the utility function:

$$
a^{\prime}=\arg \max _{a \in A} s(u, a)
$$

A successful recommender system needs to address a number of intrinsic challenges ${ }^{40}$. The news domain introduces additional complexity when compared to other areas such as books, movies or music, given its relevant temporal dimension. The news domain is intrinsically dynamic and unpredictable as there is a significant stream of stories coming up all the time. Some of these stories uncover new events, while others just report on the progress of an already published event. For this work, we focused in some specific assumptions as mentioned in García el al. ${ }^{16}$ :

- Novelty - In general, users tend to be more interested in the latest news rather than in something that happened a long time ago.

- User history - The latest news a user has read are very important to produce recommendations, because the user is intentionally showing interest on a topic or a set of topics.

- Location - Users are more interested in news related to nearby events: the closer a user is to the place of the happening, more probably this can affect him. A news recommender system should then take into account the location where the action described in the piece of news took place. In a mobile environment scenario, user location has to be frequently updated and considered.

As discussed before, hybrid recommendation systems combine two or more recommendation methodologies. In this work, the implemented hybrid recommendation algorithm combines content-based and georeferenced recommendations approaches. The final recommendation list is constructed by merging all recommendation lists resulting from each of the recommendations' approaches.

\subsubsection{Content-based recommendations}

In this work, a content-based approach is used to generate recommendations by matching short-term and long-term profiles with news articles information. 

interest

For the long-term profile, the algorithm matches the tags that have been cumulatively added to the profiles with the tags of existing news.

For the short-term profile, actions accomplished by the reader (read, like or share) are considered to have different levels of relevance. For example, if a user shares a news story on Facebook, this news is likely to have had more impact on the reader than a story that he just read without sharing it. Table 1 lists the available actions associated to its relevance weight. The higher the action weight the higher the importance of the action. For each action, a list with the $\mathrm{N}$ most similar news is created.

Table 1. Action weights

\begin{tabular}{cc}
\hline Action name & Action weight \\
\hline Read & 1 \\
Like & 2 \\
Share & 3 \\
\hline
\end{tabular}

In order to generate the final recommendation list, all the individual lists are combined. Each list is first sorted according to the action weights - higher action weights having a better position. The final list gets one element from each of the individual lists and the process keeps going until the desired length is achieved.

\subsubsection{Geo referenced recommendations}

The Georeferenced approach recommends news having the same geolocation of the mobile device.

Although the geolocation module of the mobile device is able to return very precise information that enables identifying the city or even the street name, our implementation only considers the country where the device is located. The reason for this is that news hardly have very detailed metadata schemas on location. Our approach can be refined and granularity of the location enhanced if news providers start following a strategy of more detailed description/identification of the event.

Given that Pglobal aggregates content from different newspapers in the world, geolocation is considered in two facets: geographic location (country) of the news provider and identification of the location of the event being described in the content. Geolocation information is coded using ISO 3166-1 Alpha-2 country code.

\subsubsection{Final hybrid recommendation list}

The final recommendation list is created by combining all the previous described approaches with a pre-defined fraction for each. Additionally, a priority is assigned to each of the recommendation methodologies. Whenever one of the recommendation approaches cannot fill the final list in the required percentage, the recommendations will be provided from the following recommendation methodology in the priority list. 
The system was tested with selected beta-tester users. Profiles were created and a list of recommendations that reflects all the approaches was created.

Table 2 presents an example of the information collected from a device that enables creating the user profiles. Information has been processed to reflect the approaches used for short-term and long-term recommendations. Additionally, information on the location is also presented.

For the Short-term profile field, Table 2 presents the most relevant set of stemmed terms extracted from the news with which the user has recently interacted with. The terms in bold identify the ones to which a match was found with other existing news that are then candidate to integrate the final recommendations' list.

The long-term profile section presents the list of the top 20 tags associated with the news the user read in the past.

Table 2. Example of a user profile

\begin{tabular}{cc}
\hline Geolocation & br \\
\hline & $\begin{array}{c}\text { produt, emergenci, alergi, cheg, legislativ, violenci, entregar, cheg, } \\
\text { doent, laranj, rajad, amig, deve, forn, natalidad, castel, avis, ficca, } \\
\text { lisbo, distrit, opinia, empres, burundi, joao, encenaca, miam, famili, } \\
\text { saud, integrad, gesta, farmaci, beja, teatr, port, director, alemanh, } \\
\text { integrad, sofisticad, cart, franc, espanh, horari, vesper, mandat, alun, } \\
\text { sociedad, maxim, psd, sent, politic, politic }\end{array}$ \\
\hline & PSP(5), Sociedade(5), local(4), Lisboa(4), Câmara do Porto(3), \\
Opinião(3), São João(3), Ministério Público(2), Ministério da \\
Educação(2), Violência(2), Mundo(2), Educação(2), Conflitos(2), \\
PSD(2), Meteorologia(2), França(2), Universidade Lusófona(2), \\
Universidade Lusiada(2), Moldávia(2)
\end{tabular}

Figure 5 shows an extract of the resulting recommendation list considering the user profile described. In this list, items related to each of the approaches are presented. Words in red are shown to clarify the reason that justifies the news to have been chosen as a recommendation. 

interest

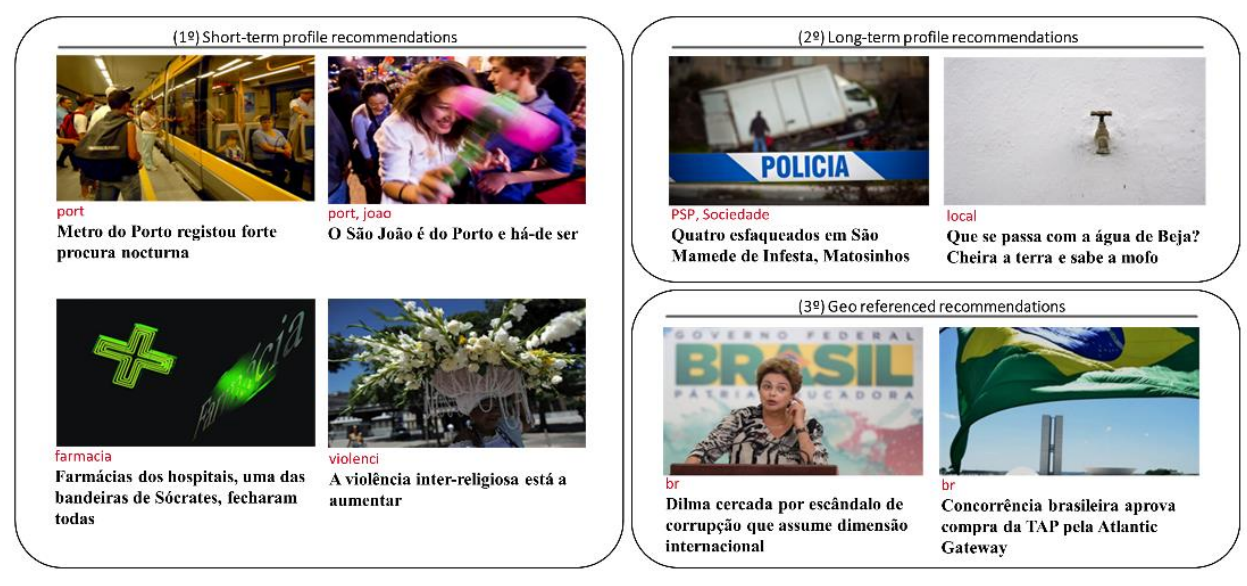

Figure 5. Recommendation list aggregating multiple recommendation approaches

\section{Experimental evaluation setup}

In order to evaluate the recommendation system, a user study was conducted by recruiting a set of volunteer subjects, with the only requirement of being readers of the newspaper, and asking them to perform two distinct tasks.

The user evaluation group consisted of 35 participants with different reading profiles: (1) seasonal readers that had only read a very small number of news; (2) average readers having an intermediate amount of news accesses; (3) intensive readers, which have a high reading historical profile and are probably the kind of reader that consumes news every day.

Figure 6 presents the distribution of the number of read news for the test population.

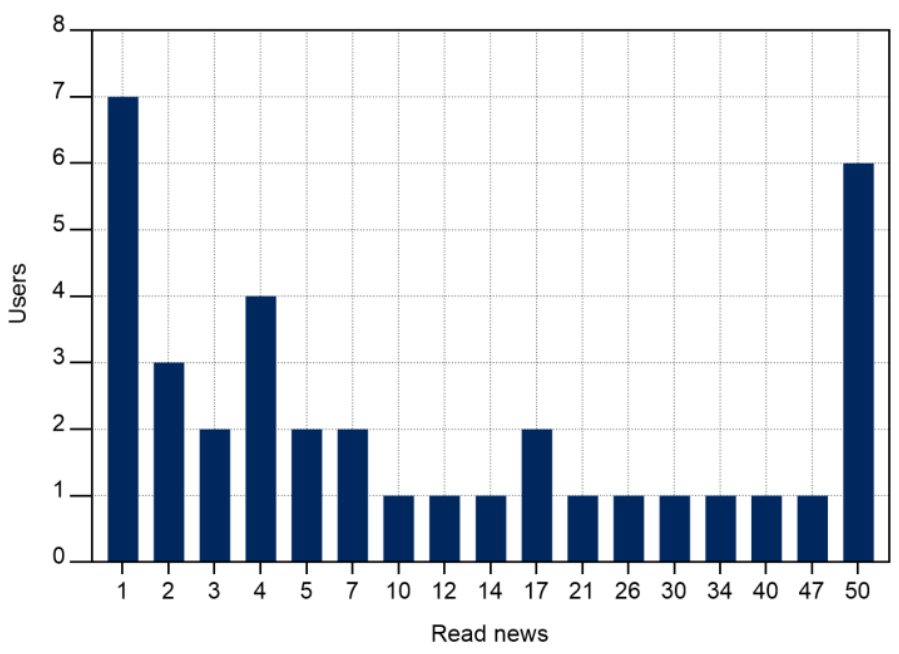

Figure 6. Distribution of the number of read news for the evaluation group 
Users were asked to perform the following evaluations and to provide their feedback:

- The first experiment consisted on presenting to the user three lists of news corresponding to 1) the output of the recommendation system, 2) the most popular news and 3) randomly selected news. Each list was composed of 5 newspaper news, horizontally aligned. For this task, the user was requested to select the list of news that best matched his preferences. The ranking position of each list was randomly generated in order to dismiss possible ranking influence. The main purpose of this experiment is to evaluate the impact of the recommendation algorithm on users' satisfaction when compared to the other two simple approaches.

- For the second evaluation task, the user was shown 12 news resulting from the 3 recommendations approaches (Geo-located, Short-term, Long-term) and asked to rate, 1 to 5, each. Each algorithm generated the same number of recommendations. The main purpose of this experiment is evaluating the effectiveness of each of the approaches on the user's satisfaction.

\section{Results and discussion}

Figure 7 and Figure 8 summarize the results obtained in the first experiment.

Figure 7(a) (resulting from the feedback of the first experiment) confirms that the list resulting from the recommendation algorithm was most often chosen, obtaining almost $50 \%$ of the total number of selections. The most popular news list comes afterwards, with $29 \%$ of the selections and finally the random list. Only a small difference is observed for these two lists, showing that not always the users enjoy popular news. Results are consistent independently of the position of the recommendation list in the experiment, as depicted in Figure 8, showing that there is no bias on the analysis.

Figure 7(b) further strengthens the good acceptance of the developed system when considering only loyal readers. Given that volunteers had a large range of reading histories, some of them with a very short record, the analysis presented includes only readers with more than 40 read news.
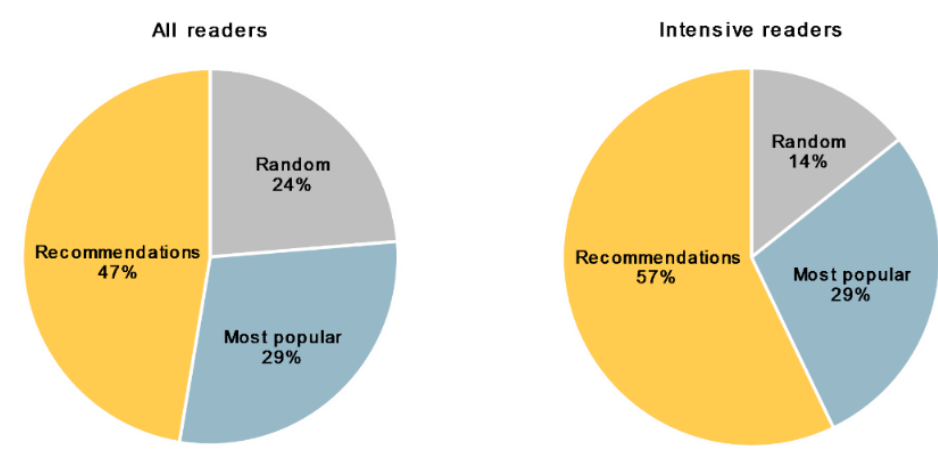

Fig. 7. User preference on news lists generated by the recommendation algorithm and two other simple approaches: (a) for all readers, (b) for readers with a significant record of accesses 

interest

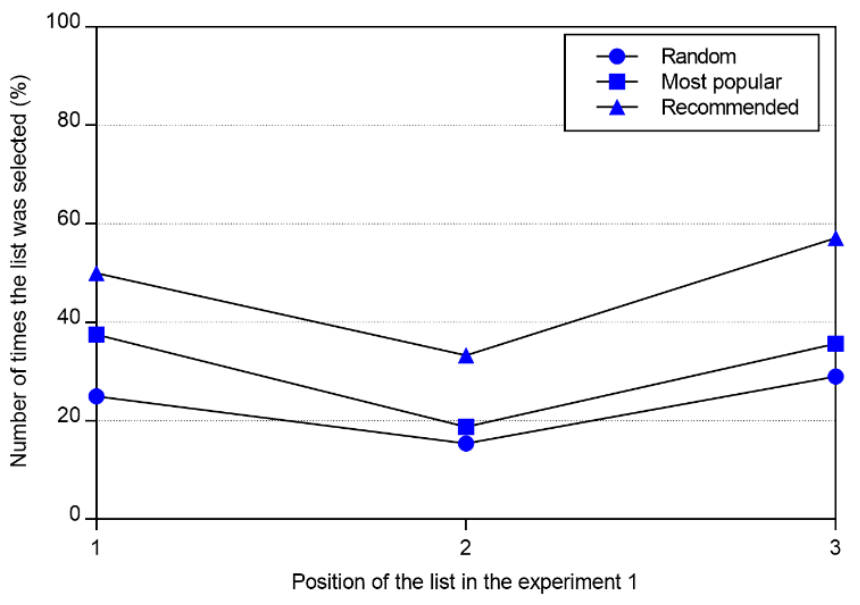

Fig. 8. Analysis of the influence of the position of the list in the user selection

These results certify the good performance of the developed recommendation approach showing that users will more likely read recommended news instead of popular news or other generic content that shows up on the screen. This is expected to contribute to the user's satisfaction and fidelity to the system since he did not have to waste too much time searching for news of his interest.

Figure 9 and Figure 10 present the results obtained by the accomplishment of task 2 . The analysis focused both on all the newsreaders who participated in this experiment and on intensive newsreaders.

In both cases, the results indicate that the short-term recommendation algorithm obtained the highest preferences (rating average). These results demonstrate users' higher interest for news similar to those ones he read recently. The low performance of the georeferenced algorithm may be due to the use of only generic information about the country where the user is located. This is a current limitation that might be improved in the future if additional information is introduced by the news provider. Another factor that might have contributed to the limited performance of the geo referenced algorithm may be related to the intrinsic limitations of the evaluation process. Ideally, this algorithm should be tested multiple times with the same user on different geographic locations so that he could truly experience the georeferenced personalization process. However, this kind of tests is very difficult to implement since the test subjects would need to perform the evaluation procedure in different countries.

Standard deviation values for the user ratings shows that for both case studies there is a similar distribution for the different recommendation algorithms. These results support the conclusion that short-term and long-term algorithms were consistently scored (low variation) with high ratings demonstrating a clear preference for those algorithms. In contrast, the geo referenced recommendation approach appears to present a high percentage of lower ratings confirming a lack of interest in this approach. In addition, these 
results also indicate that there is no significant influence of the size of the reading profile in the distribution of the ratings.

Confidence intervals of average ratings at a 95\% confidence level, for the first case study, are depicted in Figure 9 : for the short-term approach results range between 3.16 and 3.58; for long-term between 2.84 and 3.21 and for georeferenced between 2.30 and 2.70. The analyses of the records indicate that there is no significant overlap between distinct recommendation approaches. This is consistent with previous results analyses demonstrating that for the overall newsreaders population short-term recommendations should be assigned in the first ranking positions with a higher percentage of news. Recommendations provided by long-term algorithm should be presented in the intermediate position, while the georeferenced algorithm should provide a smaller number of items in the final collection.

For the second case study (Figure 10), which considers only the newsreaders with a significant record of accesses, at a 95\% confidence level, we estimate the average rating for short-term approach to be between 2.90 and 3.74, for long-term to be between 2.30 and 3.12 and for georeferenced to be between 1.99 and 2.87. Notably, in this case, the confidence intervals are wider, representing some imprecision in the results. Causes for these findings include the significantly smaller size of the population sample. Furthermore, the analysis of the records indicated that there is a more apparent overlap between preferences for distinct recommendation algorithms. This can be interpreted as an indication that short-term recommendations are less relevance for users with a more accurate reading profile. Similarly, geo referenced recommendations also appear to have increased their statistical relevance compared to previous results.

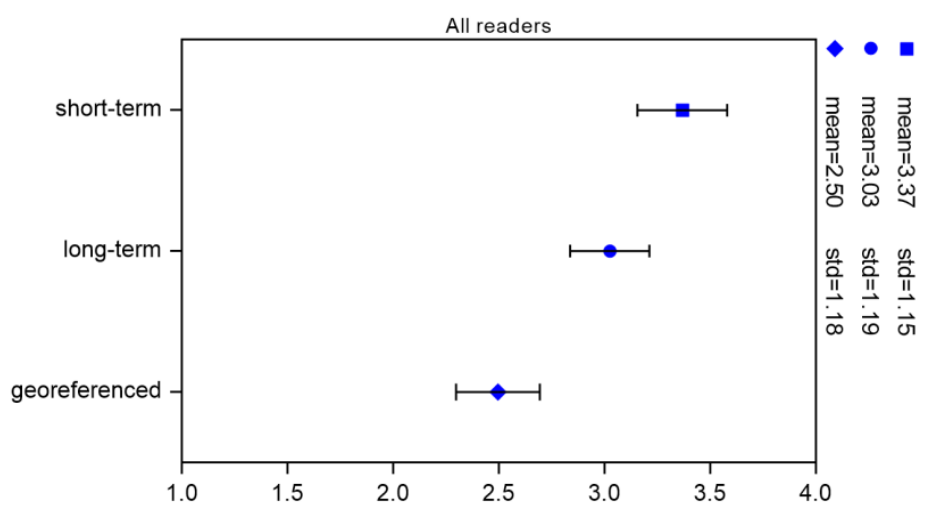

Fig. 9. Rating average at $95 \%$ of confidence level for all readers 


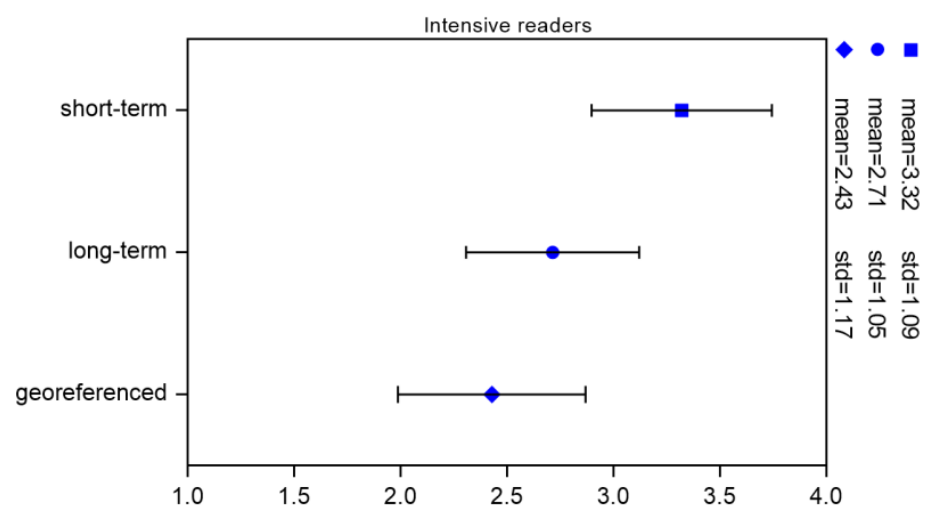

Fig. 10. Rating average at $95 \%$ of confidence level for readers with a significant record of accesses

\section{Conclusion}

The interest on online newspapers has been growing significantly over the past years. In order to present the most relevant news articles to users, different recommendation systems have been made available using various techniques in order to make access to large amounts of information more efficient.

Mobility, social networking and the large number of news providers, bring new challenges but also new opportunities to enhance access to information and improve the user experience when browsing newspapers. This paper presents a hybrid recommendation system for news in a mobile environment that takes into consideration aspects that reflect this new paradigm.

The described approach combines content-based and georeferenced recommendation methodologies. The system is able to collect information implicitly and to create shortterm and long-term user profiles that enable recommending: (1) items similar with those the user has recently interacted with (read, share or like); (2) news containing some of the terms/tags most read by the user. Additionally, the location of the mobile device is also implicitly registered in order to recommend news associated to that location.

To the best of our knowledge, this is the first implementation of a news recommendation system for mobile environments that uses the sharing and like of news in social networks to implicitly infer in a more accurate way the user preferences.

The user study acknowledges the good performance of the developed system. Moreover, the results further demonstrated that preferences are mostly influenced by recent interactions than by long last user profiles.

Future work includes evaluating the performance of our implementation using a larger set of users in a real environment. In order to evaluate the level of satisfaction with the recommendations suggested by the system, we will analyse the percentage of recommendations read by the user considering the total amount of news he read. Another experience will involve changing or switch the priority value and the percentage that each of the recommendation approaches provides to the final list and analysing the impact. With 
these analyses it will also be possible to verify how the user reading behaviour changes and try to infer what is the preferred approach.

\section{Acknowledgments}

The authors would like to thank all the participants in the project Pglobal (Nr. 2014/38592Programa Operacional Temático Factores de Competitividade/Programa Operacional do Norte, Funded by ERDF) and in particular Publico for having provided all the grounds and news that enable testing the system in a near-real environment. The work was partially supported by Project "TEC4Growth - Pervasive Intelligence, Enhancers and Proofs of Concept with Industrial Impact/NORTE-01-0145-FEDER-000020", under Research Line FourEyes, financed by the North Portugal Regional Operational Programme (NORTE 2020), under the PORTUGAL 2020 Partnership Agreement, and through the European Regional Development Fund (ERDF).

\section{References}

1. G. Adomavicius and A. Tuzhilin, Toward the next generation of recommender systems: a survey of the state-of-the-art and possible extensions, IEEE Transactions on Knowledge and Data Engineering 17(6) (2005) 734-749. doi:10.1109/TKDE.2005.99.

2. J. Ahn, P. Brusilovsky, J. Grady, D. He and S.Y. Syn, Open User Profiles for Adaptive News Systems: Help or Harm?, in Proc. of the 16th International Conference on World Wide Web (New York, USA, 2007), pp. 11-20.

3. D. Billsus and M.J. Pazzani, User Modeling for Adaptive News Access, User Modeling and User-Adapted Interaction 10(2-3) (2000) 147-180, doi:10.1023/A:1026501525781.

4. D. Billsus and M. J. Pazzani, A Hybrid User Model for News Story Classification, in Proc. of the Seventh International Conference on User Modeling (Secaucus, NJ, 1999), pp. 99-108.

5. R. Blanco, D. Ceccarelli, C. Lucchese, R. Perego and F. Silvestri, You Should Read This! Let Me Explain You Why: Explaining News Recommendations to Users, in Proc. of the 21st ACM International Conference on Information and Knowledge Management (Maui, Hawaii, 2012), pp. 1995-1999.

6. H. L. Borges and A. C. Lorena, A Survey on Recommender Systems for News Data, in Smart Information and Knowledge Management, eds. E. Szczerbicki and N.T. Nguyen, (Springer Berlin Heidelberg, 2010), pp. 129-151.

7. M. Capelle, F. Frasincar, M. Moerland and F. Hogenboom, Semantics-based News Recommendation, in Proc. of the 2Nd International Conference on Web Intelligence, Mining and Semantics (Craiova, Romania, 2012), pp. 27:1-27:9.

8. T. Chen, W. L. Han, H. D. Wang, Y. X. Zhou, B. Xu and B. Y. Zang, Content Recommendation System Based on Private Dynamic User Profile, in Proc. of International Conference on Machine Learning and Cybernetics (Hong Kong, Japan, 2007), pp. 2112-2118

9. C. -M. Chen and Y. -C. Yang, An Intelligent Mobile Location-Aware Book Recommendation System with Map-Based Guidance That Enhances Problem-Based Learning in Libraries in Advances in Neural Network Research and Applications, eds. Z. Zeng and J. Wang (Springer Berlin Heidelberg, 2010), pp. 853-860.

10. M. Claypool, A. Gokhale, T. Miranda, P. Murnikov, D. Netes and M. Sartin, Combining Content-Based and Collaborative Filters in an Online Newspaper, in ACM SIGIR '99 Workshop on Recommender Systems (Berkley, CA, 1999). 
11. B. Cui and X. Chen, An Online Book Recommendation System Based on Web Service, in Sixth International Conference on Fuzzy Systems and Knowledge Discovery (Tianjin, China, 2009), pp. 520-524.

12. A. S. Das, M. Datar, A. Garg and S. Rajaram, Google News Personalization: Scalable Online Collaborative Filtering, in Proc. of the 16th International Conference on World Wide Web (Banff, Alberta, 2007), pp. 271-280.

13. F. Frasincar, J. Borsje and L. Levering, A Semantic Web-Based Approach for Building Personalized News Services, International Journal of E-Business Research 5(3) (2009) 35-53, doi:10.4018/jebr.2009082103.

14. E. Gabrilovich, S. Dumais and E. Horvitz, Newsjunkie: Providing Personalized Newsfeeds via Analysis of Information Novelty, in Proc. of the 13th International Conference on World Wide Web (New York, USA, 2004), pp. 482-490.

15. F. Gao, Y. Li, L. Han and J. Ma, InfoSlim: An Ontology-Content Based Personalized Mobile News Recommendation System, in Proc. of the 5th International Conference on Wireless Communications, Networking and Mobile Computing (Beijing, China, 2009), pp. 1-4.

16. A. M. García, J. M. Álvarez-Rodríguez, J. E. Labra-Gayo and M. Martínez-Merino, Towards a journalist-based news recommendation system: The Wesomender approach, Expert Systems with Applications 40(17) (2013) 6735-6741, doi:10.1016/j.eswa.2013.06.032.

17. F. Garcin, C. Dimitrakakis and B. Faltings, Personalized News Recommendation with Context Trees, in Proc. of the 7th ACM Conference on Recommender Systems (Hong Kong, China, 2013), pp. 105-112.

18. F. Garcin, K. Zhou, B. Faltings and V. Schickel, Personalized News Recommendation Based on Collaborative Filtering, in Proc. of the 2012 IEEE/WIC/ACM International Conferences on Web Intelligence and Intelligent Agent Technology (WI-IAT) (Macau, China, 2012), pp. 437441.

19. A. L. Garrido, M. G. Buey, S. Ilarri, I. Fũrstner and L. Szedmina, KGNR: A knowledge-based geographical news recommender, in 2015 IEEE 13th International Symposium on Intelligent Systems and Informatics (Subotica, Serbia, 2015), pp. 195-198.

20. F. Goossen, W. IJntema, F. Frasincar, F. Hogenboom and U. Kaymak, News Personalization Using the CF-IDF Semantic Recommender, in Proc. of the International Conference on Web Intelligence, Mining and Semantics (Sogndal, Norway, 2011), pp. 10:1-10:12.

21. A. Gupta and K. Singh, Location based personalized restaurant recommendation system for mobile environments, in Proc. of the 2013 International Conference on Advances in Computing, Communications and Informatics (ICACCI), (Mysore, India, 2013), pp. 507-511.

22. P. Hoffmann, A. Kaczmarek, P. Spaleniak and B. Kostek, Music Recommendation System, Journal of Telecommunications and Information Technology 2(2014) 59-69.

23. W. IJntema, F. Goossen, F. Frasincar and F. Hogenboom, Ontology-based News Recommendation, in Proc. of the 2010 EDBT/ICDT Workshops (Lausanne, Switzerland, 2010), pp. 16:1-16:6.

24. T. Joachims, D. Freitag and T. Mitchell, WebWatcher: A Tour Guide for the World Wide Web, in Proc. of the International Joint Conference on Artificial Intelligence, (San Francisco, USA, 2007), pp. 770-775.

25. N. Jonnalagedda and S. Gauch, Personalized News Recommendation Using Twitter, in Proc of the 2013 IEEE/WIC/ACM International Joint Conferences on Web Intelligence (WI) and Intelligent Agent Technologies (IAT) (Atlanta, GA, 2013), pp. 21-25. 
26. T. Kamba, H. Sakagami, Y. Koseki, ANATAGONOMY: a personalized newspaper on the World Wide Web, International Journal of Human-Computer Studies 46(6) (1997) 789-803. doi:10.1006/ijhc.1996.0113.

27. D. Kelly, Implicit Feedback: Using Behavior to Infer Relevance in New Directions in Cognitive Information Retrieval, eds. A. Spink and C. Cole (Springer Netherlands, 2005), pp. 169-186.

28. J. A. Konstan, B. N. Miller, D. Maltz, J. L. Herlocker, L. R. Gordon and J. Riedl, Grouplens: Applying collaborative filtering to usenet news, Communications of ACM 40(3) (1997) 77-87.

29. H. -J. Lai, T. -P. Liang and Y. C. Ku., Customized Internet News Services Based on Customer Profiles, in Proc. of the 5th International Conference on Electronic Commerce (Pittsburgh, Pennsylvania, 2003), pp. 225-229.

30. H. J. Lee and S. J. Park, MONERS: A news recommender for the mobile web, Expert Systems with Applications 32(1) (2007) 143-150, doi:10.1016/j.eswa.

31. A. Lehtiniemi and J. Holm, Easy Access to Recommendation Playlists: Selecting Music by Exploring Preview Clips in Album Cover Space, in Proc. of the 10th International Conference on Mobile and Ubiquitous Multimedia (Beijing, China, 2011), pp. 94-99.

32. L. Li, L. Zheng, F. Yang and T. Li, Modeling and broadening temporal user interest in personalized news recommendation, Expert Systems with Applications 41(7) (2014) 31683177, doi:10.1016/j.eswa.2013.11.020.

33. L. Li, L. Zheng and T. Li, LOGO: A Long-short User Interest Integration in Personalized News Recommendation, in Proc. of the Fifth ACM Conference on Recommender Systems (Chicago, Illinois, 2011), pp. 317-320.

34. J. Liu, P. Dolan and E.R. Pedersen, Personalized News Recommendation Based on Click Behavior, in Proc. of the 15th International Conference on Intelligent User Interfaces (Hong Kong, China, 2010), pp. 31-40.

35. P. Lops, M. de Gemmis and G. Semeraro, Content-based Recommender Systems: State of the Art and Trends in Recommender Systems Handbook, eds. F. Ricci, L. Rokach, B. Shapira and P.B. Kantor (Springer US, 2011), pp. 73-105.

36. L. Martinez, R.M. Rodriguez and M. Espinilla, REJA: A Georeferenced Hybrid Recommender System for Restaurants, in Proc. of the IEEE/WIC/ACM International Joint Conferences on Web Intelligence and Intelligent Agent Technologies (Milan, Italy, 2009), pp. 187-190.

37. K. Meehan, T. Lunney, K. Curran and A. McCaughey, Context-aware intelligent recommendation system for tourism, in Proc. of the 2013 IEEE International Conference on Pervasive Computing and Communications Workshops (San Diego, CA, 2013), pp. 328-331.

38. Osiński, An Algorithm for Clustering of Web Search Results, thesis, Poznań University of Technology (2003).

39. Osiński and D. Weiss, User and Developer Manual for version 3.14.0-SNAPSHOT (2016), http://download.carrot2.org/head/manual/index.html.

40. Ö. Özgöbek, J. A. Gulla and R. C. Erdur, A Survey on Challenges and Methods, in Proc. of the 10th International Conference on Web Information Systems and Technologies (Barcelona, Spain, 2014), pp. 278-285.

41. F. Reischach, F. Michahelles and A. Schmidt, The Design Space of Ubiquitous Product Recommendation Systems, in Proc. of the 8th International Conference on Mobile and Ubiquitous Multimedia (Cambridge, United Kingdom, 2009), pp. 2:1-2:10. 
42. P. Resnick, N. Iacovou, M. Suchak, P. Bergstrom and J. Riedl, GroupLens: An Open Architecture for Collaborative Filtering of Netnews, in Proc. of the 1994 ACM Conference on Computer Supported Cooperative Work (Chapel Hill, North Carolina, 1994), pp. 175-186.

43. K. Schouten, P. Ruijgrok, J. Borsje, F. Frasincar, L. Levering and F. Hogenboom, A semantic web-based approach for personalizing news, in Proc. of the 2010 ACM Symposium on Applied Computing (Sierre, Switzerland, 2010), p. 854.

44. B. Shapira, P. Shoval, N. Tractinsky and J. Meyer, ePaper: A personalized mobile newspaper, Journal of the American Society for Information Science and Technology 60(11) (2009) 23332346, doi:10.1002/asi.21172.

45. M. Soares and P. Viana, TV Recommendation and Personalization Systems: Integrating Broadcast and Video On demand Services. Advances in Electrical and Computer Engineering, 14(1) (2014) 115-120, doi:10.4316/AECE.2014.01018.

46. M. Soares and P. Viana, Tuning metadata for better movie content-based recommendation systems, Multimed Tools Appl. 74(17) (2015) 7015-7036, doi:10.1007/s11042-014-1950-1.

47. G. D. Solomou, A. K. Kalou, D. A. Koutsomitropoulos and T. S. Papatheodorou, A Mashup Personalization Service Based on Semantic Web Rules and Linked Data in Proc. of 2011 Seventh International Conference on Signal-Image Technology and Internet-Based Systems (SITIS) (Dijon, France, 2011), pp. 89-96.

48. M. Tavakolifard, J. A. Gulla, K. C. Almeroth, J. E. Ingvaldesn, G. Nygreen and E. Berg, Tailored News in the Palm of Your Hand: A Multi-perspective Transparent Approach to News Recommendation, in Proc. of the 22Nd International Conference on World Wide Web (Rio de Janeiro, Brazil, 2013), pp. 305-308.

49. P. Viana and M. Soares, A Hybrid Recommendation System for News in a Mobile Environment, in Proc. of the 6th International Conference on Web Intelligence, Mining and Semantics (Nîmes, France, 2016), pp. 3:1-3:9.

50. Y. Xiao, P. Ai, C. h Hsu, H. Wang and X. Jiao, Time-ordered collaborative filtering for news recommendation, China Communications 12(12) (20015) 53-62, doi:10.1109/CC.2015. 7385528.

51. F. Yang and Z.-M. Wang, A Mobile Location-based Information Recommendation System Based on GPS and WEB2.0 Services, W. Trans. on Comp. 8(4) (2009) 725-734. 